\title{
Safety and effectiveness of pembrolizumab combined with paclitaxel and cisplatin as neoadjuvant therapy followed by surgery for locally advanced resectable (stage III) esophageal squamous cell carcinoma: a study protocol for a prospective, single-arm, single-center, open-label, phase-Il trial (Keystone-001)
}

\author{
Xiaobin Shang ${ }^{1 \# \wedge}$, Gang Zhao ${ }^{2 \#}$, Fei Liang ${ }^{3 \#}$, Chen Zhang ${ }^{1 \#}$, Weihong Zhang ${ }^{4}$, Liang Liu ${ }^{4}$, Runmei Li $^{4}$, \\ Xiaofeng Duan ${ }^{1}$, Zhao Ma ${ }^{1}$, Jie Yue ${ }^{1}$, Chuangui Chen ${ }^{1}$, Bin Meng ${ }^{2}$, Xiubao Ren ${ }^{4}$, Hongjing Jiang ${ }^{1}$ \\ ${ }^{1}$ Department of Minimally Invasive Esophageal Surgery, Key Laboratory of Cancer Prevention and Therapy, National Clinical Research Center for \\ Cancer, Tianjin Medical University Cancer Institute and Hospital, Tianjin, China; ${ }^{2}$ Department of Pathology, Key Laboratory of Cancer Prevention \\ and Therapy, National Clinical Research Center for Cancer, Tianjin Medical University Cancer Institute and Hospital, Tianjin, China; ${ }^{3}$ Department \\ of Biostatistics, Zhongshan Hospital, Fudan University, Shanghai, China; ${ }^{4}$ Department of Immune Oncology, Key Laboratory of Cancer Prevention \\ and Therapy, National Clinical Research Center for Cancer, Tianjin Medical University Cancer Institute and Hospital, Tianjin, China \\ \#These authors contributed equally to this work. \\ Correspondence to: Hongjing Jiang. Department of Minimally Invasive Esophageal Surgery, Key Laboratory of Cancer Prevention and Therapy, \\ National Clinical Research Center for Cancer, Tianjin Medical University Cancer Institute and Hospital, Tiyuanbei, Huanhuxi Rd., Hexi \\ District, Tianjin 300060, China. Email: jianghongjing@tmu.edu.cn; Xiubao Ren. Department of Immune Oncology, Key Laboratory of Cancer \\ Prevention and Therapy, National Clinical Research Center for Cancer, Tianjin Medical University Cancer Institute and Hospital, Tianjin, China. \\ Email: renxiubao@tjmuch.com; Bin Meng. Department of Pathology, Key Laboratory of Cancer Prevention and Therapy, National Clinical \\ Research Center for Cancer, Tianjin Medical University Cancer Institute and Hospital, Tianjin, China. Email: mbinca@163.com.
}

Background: Esophageal squamous cell carcinoma (ESCC) is one of the most refractory malignant tumors. Esophageal cancer (EC) is a malignant tumor with a high incidence worldwide, and over 50\% of EC cases occur in China. Under the National Comprehensive Cancer Network (NCCN) guidelines, concurrent chemoradiotherapy is the only standard neoadjuvant treatment for locally advanced ESCC. In the first-line treatment of advanced ESCC, the efficacy of immune checkpoint inhibitors (ICIs) combined with systemic chemotherapy is significantly better than that of chemotherapy alone. Paclitaxel and cisplatin (TP), as one of the neoadjuvant chemotherapy regimens for locally advanced ESCC, have been widely used in China in recent years. ICIs combined with TP as neoadjuvant therapy seems promising.

Methods: This is an open-label, single-arm, single-center, phase-II trial. Locally advanced resectable (stage III) ESCC patients who have not undergone previous systemic treatments will be enrolled in this study. All the subjects will intravenously receive 3 cycles of pembrolizumab (200 $\mathrm{mg})$ on day 1, paclitaxel $\left(135 \mathrm{mg} / \mathrm{m}^{2}\right)$ on day 2 , and cisplatin $\left(20 \mathrm{mg} / \mathrm{m}^{2}\right)$ on days $2-4$, every 3 weeks. After an efficacy evaluation, the subjects will undergo Da Vinci robot-assisted radical resection. If the postoperative pathologic results do not reveal a complete response, pembrolizumab will be administrated for at least 6 cycles as an adjuvant therapy with the same usage as before. The primary endpoints are the major pathological response and safety. The secondary endpoints include the objective response rate (ORR), overall survival (OS), disease-free survival (DFS), the R0 resection rate, and perioperative complications. The exploratory endpoint is to examine the correlation between related biomarkers and tumor responses to this neoadjuvant treatment regimen.

Discussion: This trial is the first enrolled study to evaluate the safety and efficacy of pembrolizumab

$\wedge$ ORCID: 0000-0002-2211-5496. 
combined with TP as neoadjuvant therapy for locally advanced ESCC. Currently, under the NCCN guidelines, neoadjuvant chemoradiotherapy (nCRT) is the only recommended treatment for locally advanced ESCC. This phase-II study will provide preliminary evidence of the efficacy of pembrolizumab combined with TP as novel neoadjuvant therapy for patients with locally advanced ESCC.

Trial Registration: Clinicaltrials.gov (Identifier: NCT04389177).

Keywords: Esophageal squamous cell carcinoma (ESCC); locally advanced; pembrolizumab; neoadjuvant therapy

Submitted Dec 22, 2021. Accepted for publication Feb 21, 2022.

doi: 10.21037/atm-22-513

View this article at: https://dx.doi.org/10.21037/atm-22-513

\section{Introduction}

Esophageal cancer (EC) is a malignant tumor with a high incidence worldwide, and more than $50 \%$ of EC cases occur in China (1). In 2015, there were 477,900 new cases of EC in China (2). Esophageal squamous cell carcinoma (ESCC) only accounts for $30-40 \%$ of EC cases in the West, but accounts for about $95 \%$ of EC cases in China (3). EC in China has distinct clinicopathological characteristics to EC in the West.

The NCCN guidelines and the Chinese Society of Clinical Oncology (CSCO) guidelines both recommend nCRT as the standard neoadjuvant treatment for locally advanced ESCC. Neoadjuvant chemotherapy (nCT) is also recommended by Chinese and Japanese guidelines. The CROSS (4) and NEOCRTEC5010 (5) studies established neoadjuvant chemoradiotherapy (nCRT), which has a high pathological complete response (pCR) rate of more than $40 \%$, as the standard treatment for locally advanced ESCC; however, the long-term outcomes of ESCC patients are still not satisfactory. The 5 -year overall survival (OS) rate of patients treated with nCRT followed by surgery is about $50 \%$, and the incidence of locoregional or distant metastasis is also high (6). Additionally, it is likely that radiotherapy would increase the operative difficulties, and perioperative complications, such as radiation pneumonia and esophageal fistulas, would lead to a further decline in the quality of life of patients and a worse prognosis. Thus, a novel mode of neoadjuvant therapy that not only improves tumor response and survival but also has a reduced impact on surgery and good safety needs to be further established.

Pembrolizumab, a checkpoint inhibitor that targets programmed death-1 (PD-1), has been proven to have efficacy in the treatment of advanced ESCC (7-9). The Keynote-590 study (10) recommended that pembrolizumab combined with chemotherapy [5-fluorouracil and cisplatin
(FP)] be used as the standard first-line treatment for advanced or metastatic ESCC. However, the question arises: if pembrolizumab continues to be used as a neoadjuvant treatment before surgery, what effect will it achieve? Paclitaxel-based chemotherapy has obvious advantages in the treatment of ESCC patients $(11,12)$, and is already widely used in clinics in China. Taken together, pembrolizumab combined with $\mathrm{TP}$ appear to be a very promising therapeutic modality for ESCC.

At present, there is no biomarker for predicting the efficacy of pembrolizumab combined with chemotherapy, and the programmed death-ligand 1 (PD-L1) expression [Combined Positive Score (CPS)] can not accurately predict the efficacy of immunotherapy for ESCC.

Thus, we designed the Keystone-001 study and plan to recruit 50 subjects with locally advanced (stage III) ESCC to explore the efficacy and safety of pembrolizumab combined with TP as a neoadjuvant therapy followed by surgery. Additionally, PD-L1 expression (CPS), microsatellite instability (MSI), peripheral blood $\mathrm{T}$ cell subsets, and tumor-infiltrating lymphocytes (TILs) as potential biomarkers to predict the efficacy of the novel therapy.

\section{Methods}

\section{Study design}

Keystone-001 is a prospective, single-arm, single-center, open-label, phase-II study. The total number of recruits is 50 cases. Estimated Primary Completion Date: May 15, 2022; Estimated Study Completion Date: December 31, 2024

The study protocol was approved by the Ethics Committee of Tianjin Medical University Cancer Institute and Hospital (Nos. E2020101 and E2020566). All the subjects will participate in the current clinical trial on a voluntary basis and will sign the informed consent form. 
The present clinical trial conforms to the Good Clinical Practice (GCP) released by SFDA (Sino Food and Drug Administration), and the relevant laws and regulations.

\section{Inclusion and exclusion criteria}

Patients who have been histologically confirmed to have locally advanced ESCC (cT3N1M0, cT1-3N2M0, and stage III) with a performance status score of 0 to 1 on the Eastern Cooperative Oncology Group Performance Status (ECOG PS) and proper organ function will be eligible to participate in this trial. The main exclusion criteria are previous chemo-, immune- or radiation therapy. The full inclusion and exclusion criteria are shown in Table 1.

\section{Objectives}

\section{Primary objectives}

The primary objective of this study is to evaluate the safety and major pathologic response (MPR) of neoadjuvant therapy (pembrolizumab plus TP) in patients with locally advanced ESCC who have not undergone any previous systemic treatments.

\section{Secondary objectives}

The secondary objectives of this study are the pCR, objective response rate (ORR), disease-free survival (DFS), OS, $\mathrm{R} 0$ resection rate, and lymph-node derating rate for pembrolizumab combined with TP chemotherapy in patients with locally advanced ESCC who have not undergone any previous systemic treatments. The safety evaluation will include the median cycles of the neoadjuvant therapy, any adverse events (AEs) related to neoadjuvant therapy, and perioperative complications. The quality of life and nutritional status of patients will be assessed using questionnaires [i.e., the EORTC QLQ (European Organization for Research and Treatment of Cancer quality of life)-C30, EORTC QLQ-OES18, and PG-SGA (Patient-Generated Subjective Global Assessment)].

Safety assessment results mainly refer to AEs related to neoadjuvant therapy, including rash, pruritus, leukopenia, thrombocytopenia, nausea, vomiting, diarrhea, and whether it affects surgical treatment. Efficacy evaluation outcomes includes MPR, pCR, ORR, DFS, OS, R0 resection rate, and lymph-node derating rate.

\section{Exploratory objectives}

The exploratory purpose of this study is to further identify the predictive biomarkers related to the efficacy of neoadjuvant therapy. The following patient parameters are considered potential predictive biomarkers, and their correlations with tumor responses will be explored:

(I) In the tumor issues: the expression of PD-L1 (CPS score), MSI, and the number of TILs;

(II) In the peripheral blood: $\mathrm{T}$ cell subsets [the absolute counts of cluster of differentiation (CD) 3, CD4, and CD8], the tumor markers, Epidermal Growth Factor (EGF), Platelet-Derived Growth Factor BB (PDGF-BB), Brain Derived Neurotrophic Factor (BDNF), Macrophage Inflammatory Protein-1 Beta (MIP-1 $\beta$ ), Interleukin-8 (IL-8), and Macrophage Migration Inhibitory Factor (MIF).

\section{Baseline evaluation}

The baseline status assessment will be carried out within 2 weeks of the first treatment. At this stage, specific information about each tumor will be collected to provide a baseline level. The related procedures include testing for tumor markers [squamous cell carcinoma antigen (SCC)], imaging evaluations [via computed tomography (CT)], and PD-L1 protein expression levels (CPS scores), MSI, circulating tumor cells, the number of TILs, the absolute counts of CD3, CD4, and CD8 T cells, the diversity of TCR, the cloning of TCR in tumor tissues, and the levels of EGF, PDGF-BB, BDNF, MIP-1 $\beta$, IL-8, and MIF.

\section{Treatment and follow-up}

After successfully completing the screening phase and confirming patient eligibility, the treatment will begin. On day 1 , a fixed dose of $200 \mathrm{mg}$ of pembrolizumab will be administered via an intravenous drip. Pembrolizumab will be administered every 3 weeks for a total of 3 cycles. The last infusion will occur in week 7 . Neoadjuvant chemotherapy will start on day 2. Paclitaxel $\left(135 \mathrm{mg} / \mathrm{m}^{2}\right)$ will be administered on day 2 via an intravenous drip, 1 time/ 3 weeks, for a total of 3 cycles, and cisplatin $\left(20 \mathrm{mg} / \mathrm{m}^{2}\right)$ will be administered on days 2-4 via an intravenous drip, 1 time/3 weeks, for a total of 3 cycles (see Table 2).

The imaging will be performed to provide a baseline before the start of treatment, and will be repeated after the completion of neoadjuvant treatment and before the da Vinci radical resection of EC is completed to rule out progression or systemic disease. If systemic disease is detected, the radical resection of EC will not 
Table 1 Inclusion and exclusion criteria

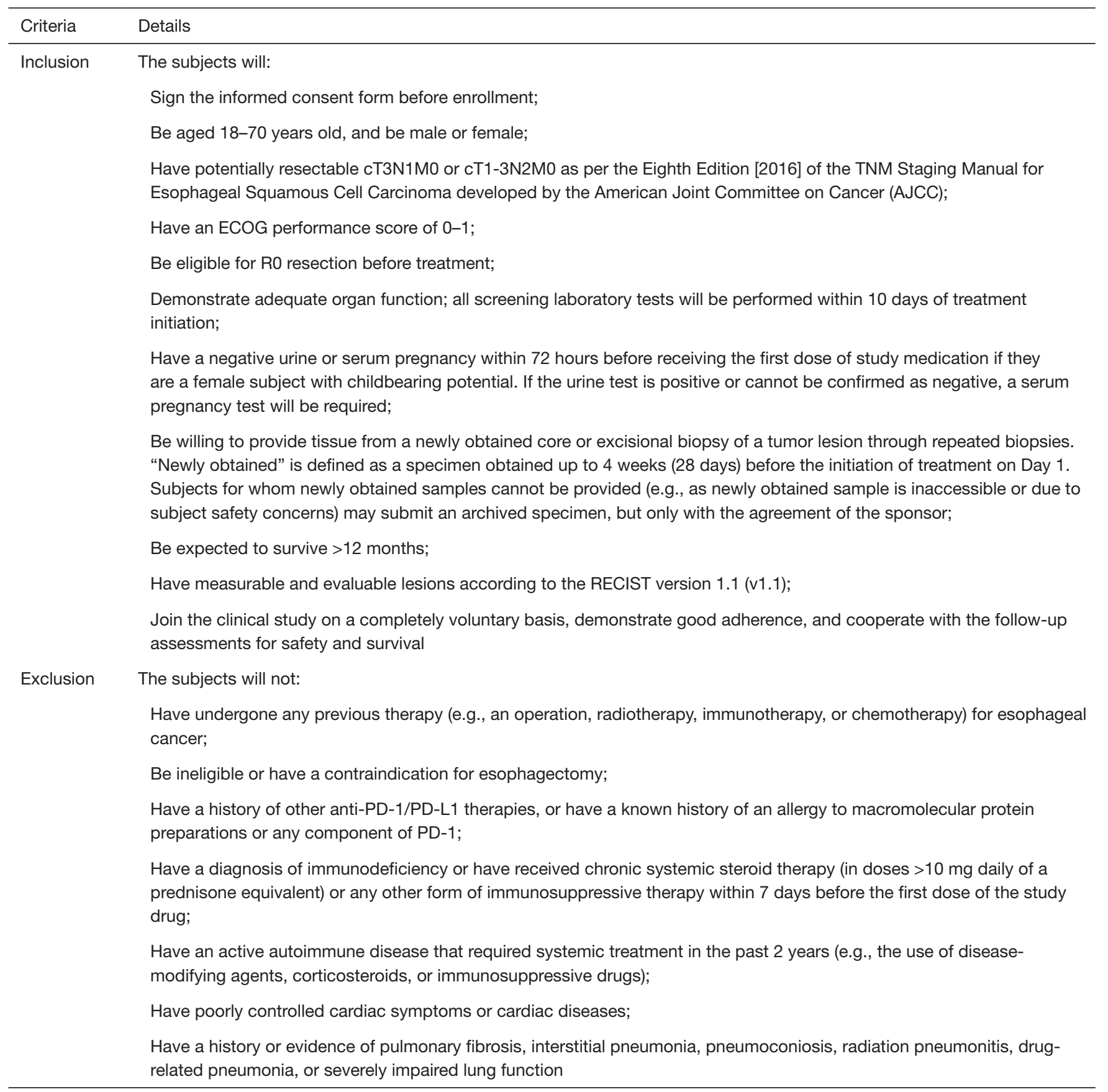

ECOG, Eastern Cooperative Oncology Group; PD-1, programmed death-1; PD-L1, programmed death ligand-1.

Table 2 Medical treatment of the patients

\begin{tabular}{lcccc}
\hline Drug & Dose & Administration frequency & Administration pathway & Treatment cycle \\
\hline Pembrolizumab & $200 \mathrm{mg}$ & Once every 3 weeks & Intravenous injection & Day 1 of each cycle \\
Paclitaxel & $135 \mathrm{mg} / \mathrm{m}^{2}$ & Once every 3 weeks & Intravenous injection & Day 2 of each cycle \\
Cisplatin & $80 \mathrm{mg} / \mathrm{m}^{2}$ & Once every 3 weeks & Intravenous injection & Day 2-4 of each cycle \\
\hline
\end{tabular}


be performed for the purpose of curing the patient. The surgery will be performed within 11 to 13 weeks, 4-6 weeks after completion of neoadjuvant therapy and will include EC resection and standardized lymph node dissection (McKeown esophagectomy). The procedure for standard lymph node dissection is precisely defined in the Standard Operation Procedure (SOP), and will include the upper mediastinal lymph nodes, intrathoracic paraesophageal lymph nodes, and abdominal lymph nodes. If the postoperative pathological results do not indicate a complete pathological response, the patient will continue to be given pembrolizumab, at the same dosage and schedule as before the operation.

The follow-up period will start after all the treatments have ended and will last for 3 years. A schematic outline of the treatment plan is shown in Figure 1.

The follow-up is based on National Comprehensive Cancer Network (NCCN) guidelines. After the completion of the radical EC surgery and discharge from hospital, visits will be conducted every 3 months to assess survival status, the laboratory test results, AEs, and the ECOG score, and perform ultrasounds of the neck and upper abdomen, and CT scans of the chest, abdomen, and pelvis. Quality of life and nutritional status will be assessed using questionnaires (i.e., the EORTC QLQ-C30, EORTC QLQ-OES18, and PG-SGA) before and after the neoadjuvant therapy and every 3 months after surgery. Figure 2 shows a detailed flow chart of the minimum evaluation during the treatment process.

\section{Outcome measures}

\section{Clinical efficacy assessment}

The following indicators are necessary to evaluate clinical efficacy:

(I) MPR: MPR is defined as $\leq 10 \%$ of the residual viable tumor at the time of resection.

(II) ORR: according to RECIST 1.1, the efficacy evaluation can be divided into complete response (CR), partial response (PR), stable disease (SD), and disease progression (PD). In this trial, ORR $=\mathrm{CR}+\mathrm{PR}$, and $\mathrm{DCR}=\mathrm{CR}+\mathrm{PR}+\mathrm{SD}$. The size of the lesion (based on the imaging and physical evaluations) will be used to compare the results, and the accuracy checks will be performed in the same way throughout the trial. The ORR and DCR with $95 \%$ confidence intervals will be calculated.

(III) Survival outcome: DFS is defined as the time from the start of treatment to the first confirmation of $\mathrm{PD}$ or cancer-related death. The overall survival (OS) is from the beginning of treatment until the death of the patient. A Kaplan-Meier analysis will be conducted to estimate and determine the DFS and OS.

(IV) The R0 resection rate is defined as the percentage of successful R0 resections.

\section{Pharmacodynamic analysis}

Fisher's exact test will be used to analyze the correlations between the ORR and the potential pharmacodynamic parameters (e.g., PD-L1 expression, TIL number, TCR clones in TIL, peripheral blood T cell subsets, and peripheral blood TCR diversity). When there is a correlation between the tumor response frequency distribution graph and the level of biomarkers, a curve will be drawn. A Cox proportional hazard regression analysis will be conducted to study the relationship between the tumor responses and biomarkers.

\section{Safety assessment}

In the safety assessment, any grade of $\mathrm{AE}$ and any serious AEs (SAEs) during treatment will be observed and recorded. We will also assess the laboratory analysis results, ECG results, physical examination results, and Performance Status (PS) scores. AEs will be classified according to The National Cancer- Institute Common Terminology Criteria for Adverse Events (NCI-CTCAE) 5.0. The researchers will be responsible for implementing appropriate measures for any AEs and determining the causal relationship between the AEs and experimental drugs.

\section{AEs}

Details of AEs will be collected throughout the study period (treatment and follow-up). Details of all SAEs will be collected from the beginning of the screening period until the postoperative period. The AEs of interest include immune-mediated events, pneumonia, diarrhea/colitis, hepatitis, nephritis/renal insufficiency, rash, and endocrine events (e.g., adrenal insufficiency, hypothyroidism/ thyroiditis, and hyperthyroidism). In addition to the evaluation of all AEs through NCI-CTCAE 5.0, details of any AEs related to surgery will be collected, including perioperative complications (e.g., anastomotic leakage, blood loss, lung infection, cardiovascular events, and fever).

\section{Statistical analysis}

This trial is a single-arm trial, and is not a randomized 


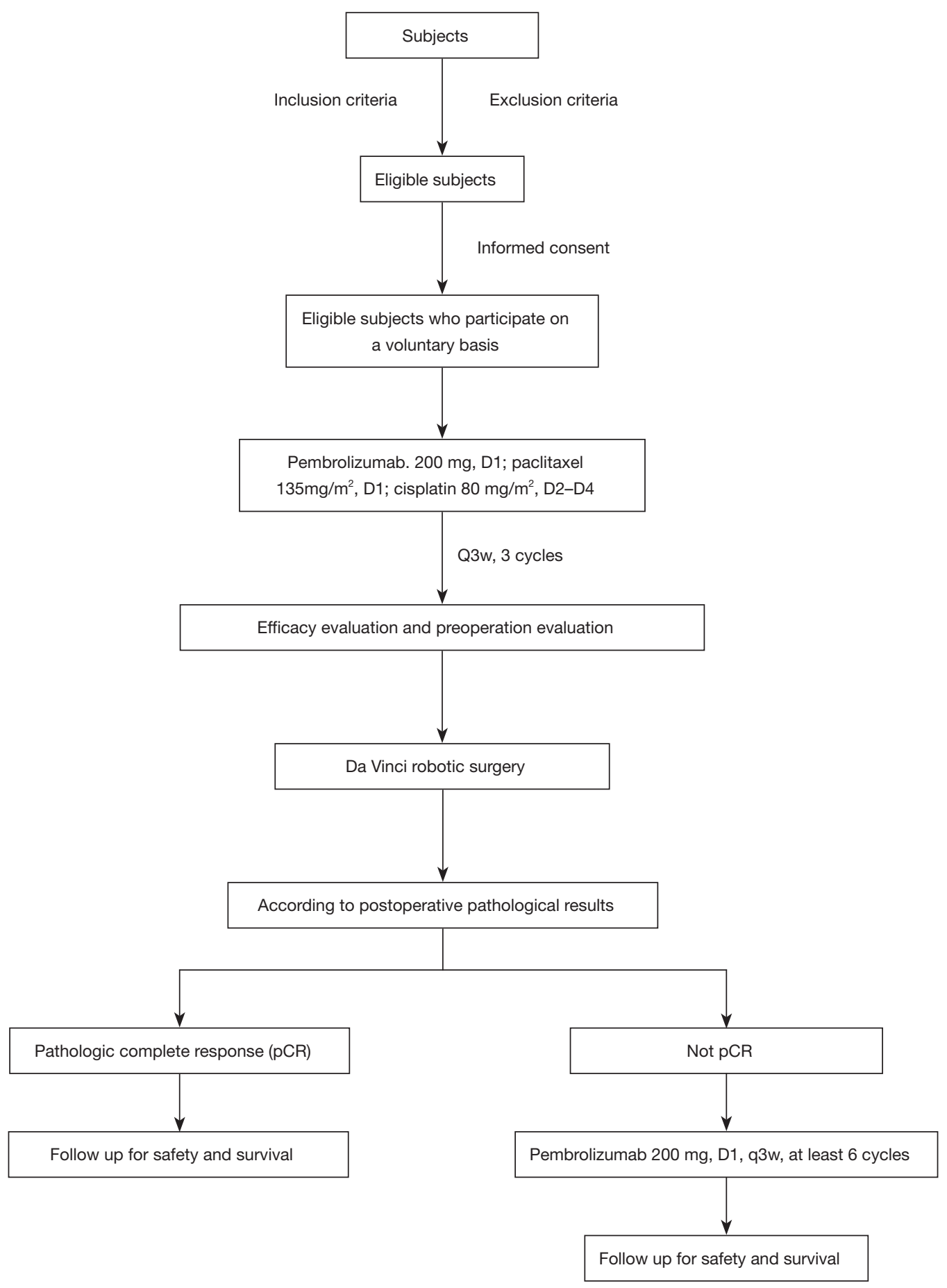

Figure 1 Study flowchart.

clinical trial. The ESCC clinical trial for which OS is the primary endpoint will take 5 years or more to complete, which limits innovation. Survival alternatives, such as pathological responses to neoadjuvant therapy, may increase the efficiency of the trial and accelerate our progress. After neoadjuvant treatment, $\leq 10 \%$ of the remaining surviving tumors, referred to here as the MPR, will meet the surrogate standard. This is due to the effects of the 


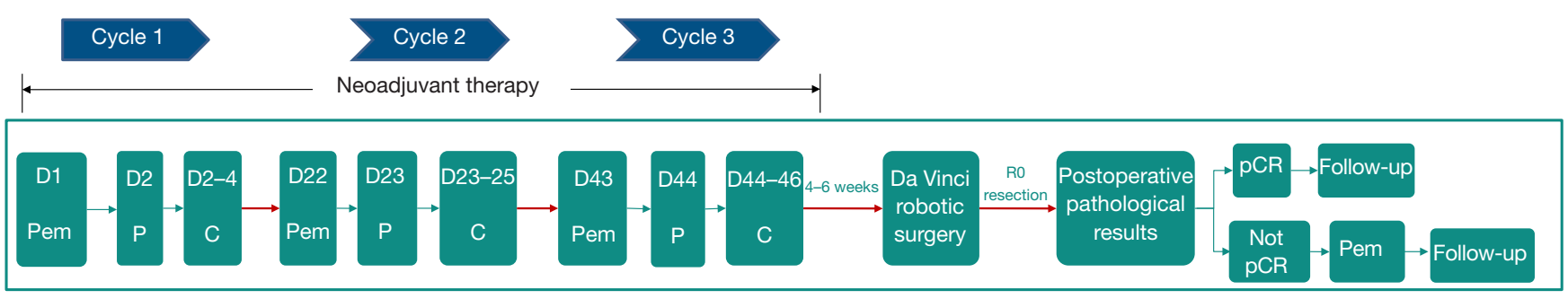

Pem, Pembrolizumab; P, Paclitaxel; C, Cisplatin; pCR, Pathologic complete response

Figure 2 The therapeutic strategy for the enrolled patients.

treatment and reflects the benefits of the treatment for survival. In a clinical trial examining the efficacy of nCRT in the treatment of clinical stages I-III EC, 42 of the 94 patients scheduled for resection showed MPR. In this study, the threshold of the MPR rate has been set to $45 \%$, and the expected value to $65 \%$, as pembrolizumab combined with TP chemotherapy will be administered in 3 doses before surgery. When the alpha error is 0.05 and the power is 0.8 , the number of patients required is 45 . If a loss to follow-up rate of $10 \%$ is set in this study, the total number of patients recruited should be limited to 50. Patients who cannot undergo surgery will be considered non-MPR.

Fisher's precise test was used to analyze the correlation between MPR and these potential pharmacodynamic parameters such as the number of TILs, T cell subsets, EGF, PDGF-BB, BDNF, MIP-1 $\beta$, IL-8, and MIF. When correlations exist, frequency distributions of tumor responses and curves of biomarker levels are depicted. Cox proportional hazards regression analysis was used to investigate the relationship between tumor response and biomarkers, respectively.

\section{Discussion}

To our knowledge, this is the first enrolled study to explore the efficacy and safety of pembrolizumab combined with TP as a neoadjuvant treatment for locally advanced resectable ESCC.

In China, $>50 \%$ of patients have locally advanced ESCC at the time of first diagnosis, and surgery is the main treatment choice for resectable ESCC (2). Unfortunately, most patients develop metastasis or recurrence within 3 years of surgery, and the 5 -year survival rate is only $20.6-34.0 \%$ (2). The poor prognosis of surgery alone for locally advanced ESCC has prompted doctors to explore various and comprehensive therapeutic modalities to improve survival. However, research has shown that postoperative adjuvant chemotherapy or radiotherapy has failed to improve the prognosis of patients with ESCC $(13,14)$. Thus, more attention needs to be paid to the use of neoadjuvant therapy in treating ESCC.

The NCCN guidelines and the Chinese Society of Clinical Oncology (CSCO) guidelines both recommend nCRT followed by esophagectomy as the standard treatment for locally advanced ESCC, which has a pCR rate of over $40 \%(4,5)$. However, improved pCR rate in nCRT did not provide a more significant long-term survival advantage than in nCT. Further, due to the superimposition of radiotherapy and chemotherapy toxicities, the difficulty of surgery and surgical complications increase as patients' compliance decrease, all of which restrict its widespread clinical application. Conversely, neoadjuvant chemotherapy (nCT) has many advantages, such as good tolerance, high compliance, and high surgical safety, which can significantly reduce patients' risk of death (15). The tumor response rate of $\mathrm{nCT}$ is lower than that of $\mathrm{nCRT}$, but there is no significant difference between nCRT and $\mathrm{nCT}$ in terms of OS, PFS, and recurrence patterns. Both Japanese and Chinese guidelines recommend " $\mathrm{nCT}+$ surgery" as the treatment for locally advanced ESCC. Thus, there is a need to establish a novel and effective treatment model that not only has a higher pCR rate, but also has a reduced impact on surgical resection and improves long-term survival.

The KEYNOTE-028 (7) and KEYNOTE-180 (8) studies confirmed that pembrolizumab has good safety and feasibility in the treatment of heavily pretreated ESCC patients. The phase-III KEYNOTE-181 study (9) found that advanced EC patients treated with pembrolizumab had a higher ORR ( $16.7 \%$ vs. $7.4 \%)$ and lower treatment-related AEs than those of standard second-line chemotherapy. As the first large, multi-center, randomized phase-III study to compare the efficacy of immunotherapy plus chemotherapy and chemotherapy only in a first-line setting for unresectable, locally advanced or metastatic EC or Siewert type I gastro- 
esophageal junction cancer, the KEYNOTE-590 (10) study demonstrated that pembrolizumab plus chemotherapy offered significant and clinically meaningful improvements in terms of OS, PFS, and the ORR compared to the placebo plus chemotherapy treatment, and the duration of response (DOR) of pembrolizumab plus chemotherapy also far exceeded that of standard chemotherapy. Further, according to the subgroup analysis, Asian ESCC patients could achieve more survival benefits than the global population. At present, pembrolizumab is the only immune checkpoint inhibitor (ICI) approved by both the American Food and Drug Administration and the National Medical Products Administration, and is recommended by both the NCCN guidelines and CSCO guidelines as the first- and secondline treatment of ESCC. Based on the good efficacy and safety of pembrolizumab, its application and exploration in neoadjuvant therapy for locally advanced ESCC have great prospects.

Given that immunotherapy combined with nCRT is very likely to increase the surgical difficulties (e.g., tissue adhesion, and edema) and perioperative complications (e.g., respiratory insufficiency or even failure caused by immunerelated and radiation pneumonitis), some studies are being conducted that combine ICIs and chemotherapy alone, such as the NICE study and NIC-ESCC2019 study. So, the questions arise: Compared to "nCRT + surgery", how effective is "pembrolizumab + $\mathrm{nCT}+$ surgery?" What is its impact on perioperative complications? To date, no research appears to have been conducted to address these questions.

Currently, TP is still the standard treatment for advanced ESCC at most centers, and has an effective rate of $19-58 \%$, and a 5 -year survival rate of $5.9-12 \%$ (16). Paclitaxel is an active agent against EC, and it has been shown to be a potent immunotherapy sensitizer $(17,18)$. In 2013, a phase-II clinical study (19) for the treatment of ESCC was conducted in Japan, which showed that the pCR rate reached $17 \%$, the ORR was $64.3 \%$, and the 2 -year DFS and OS were $74.5 \%$ and $88.0 \%$, respectively. These results provided evidence of the superiority of paclitaxel in the treatment of ESCC. It was then listed as the standard first-line treatment in a variety of guidelines. Thus, our study adopted the TP chemotherapy regimen widely used clinically in China as the nCT regimen.

Based on previous evidence, the addition of pembrolizumab to TP chemotherapy in ESCC has promising efficacy and acceptable toxicity, which supports the design of a this single-center, prospective, phaseII clinical study (Keystone-001). The trial is expected to initially prove the feasibility of pembrolizumab combined with TP as a neoadjuvant therapy in the treatment of locally advanced ESCC, which should provide a basis for further research.

\section{Acknowledgments}

The authors would like to offer their sincere thanks to Ms. Chen-Xi Ma from MSD medical affairs for her scientific comments on this manuscript.

Funding: This study was partially funded by the Beijing Xisike Clinical Oncology Research Foundation (No. Y-MSD2020-0346).

\section{Footnote}

Conflicts of Interest: All authors have completed the ICMJE uniform disclosure form (available at https://atm. amegroups.com/article/view/10.21037/atm-22-513/coif). The authors have no conflicts of interest to declare.

Ethical Statement: The authors are accountable for all aspects of the work in ensuring that questions related to the accuracy or integrity of any part of the work are appropriately investigated and resolved. The study protocol was approved by the Ethics Committee of Tianjin Medical University Cancer Institute and Hospital (Nos. E2020101 and E2020566). All the subjects will participate in the current clinical trial on a voluntary basis and will sign the informed consent form. The present clinical trial conforms to the Good Clinical Practice (GCP) released by SFDA (Sino Food and Drug Administration), and the relevant laws and regulations.

Open Access Statement: This is an Open Access article distributed in accordance with the Creative Commons Attribution-NonCommercial-NoDerivs 4.0 International License (CC BY-NC-ND 4.0), which permits the noncommercial replication and distribution of the article with the strict proviso that no changes or edits are made and the original work is properly cited (including links to both the formal publication through the relevant DOI and the license). See: https://creativecommons.org/licenses/by-nc-nd/4.0/.

\section{References}

1. Siegel RL, Miller KD, Jemal A. Cancer statistics, 2016. CA Cancer J Clin 2016;66:7-30. 
2. Chen $W$, Zheng R, Baade PD, et al. Cancer statistics in China, 2015. CA Cancer J Clin 2016;66:115-32.

3. Zeng H, Zheng R, Zhang S, et al. Esophageal cancer statistics in China, 2011: Estimates based on 177 cancer registries. Thorac Cancer 2016;7:232-7.

4. van Hagen P, Hulshof MC, van Lanschot JJ, et al. Preoperative chemoradiotherapy for esophageal or junctional cancer. N Engl J Med 2012;366:2074-84.

5. Yang H, Liu H, Chen Y, et al. Neoadjuvant Chemoradiotherapy Followed by Surgery Versus Surgery Alone for Locally Advanced Squamous Cell Carcinoma of the Esophagus (NEOCRTEC5010): A Phase III Multicenter, Randomized, Open-Label Clinical Trial. J Clin Oncol 2018;36:2796-803.

6. Shapiro J, van Lanschot JJB, Hulshof MCCM, et al. Neoadjuvant chemoradiotherapy plus surgery versus surgery alone for oesophageal or junctional cancer (CROSS): long-term results of a randomised controlled trial. Lancet Oncol 2015;16:1090-8.

7. Doi T, Piha-Paul SA, Jalal SI, et al. Safety and Antitumor Activity of the Anti-Programmed Death-1 Antibody Pembrolizumab in Patients With Advanced Esophageal Carcinoma. J Clin Oncol 2018;36:61-7.

8. Shah MA, Kojima T, Hochhauser D, et al. Efficacy and Safety of Pembrolizumab for Heavily Pretreated Patients With Advanced, Metastatic Adenocarcinoma or Squamous Cell Carcinoma of the Esophagus: The Phase 2 KEYNOTE-180 Study. JAMA Oncol 2019;5:546-50.

9. Kojima T, Shah MA, Muro K, et al. Randomized Phase III KEYNOTE-181 Study of Pembrolizumab Versus Chemotherapy in Advanced Esophageal Cancer. J Clin Oncol 2020;38:4138-48.

10. Sun JM, Shen L, Shah MA, et al. Pembrolizumab plus chemotherapy versus chemotherapy alone for first-line treatment of advanced oesophageal cancer (KEYNOTE-590): a randomised, placebo-controlled, phase 3 study. Lancet 2021;398:759-71.

11. Yamasaki M, Yasuda T, Yano M, et al. Multicenter randomized phase II study of cisplatin and fluorouracil plus docetaxel (DCF) compared with cisplatin and fluorouracil plus Adriamycin (ACF) as preoperative chemotherapy for resectable esophageal squamous cell carcinoma (OGSG1003). Ann Oncol 2017;28:116-20.

12. Zhang P, Xi M, Li QQ, et al. Concurrent cisplatin and 5-fluorouracil versus concurrent cisplatin and docetaxel with radiotherapy for esophageal squamous cell carcinoma: a propensity score-matched analysis. Oncotarget 2016;7:44686-94.

13. Bhansali MS, Vaidya JS, Bhatt RG, et al. Chemotherapy for carcinoma of the esophagus: a comparison of evidence from meta-analyses of randomized trials and of historical control studies. Ann Oncol 1996;7:355-9.

14. Fokas E, Rödel C. Definitive, Preoperative, and Palliative Radiation Therapy of Esophageal Cancer. Viszeralmedizin 2015;31:347-53.

15. Ando N, Kato H, Igaki $H$, et al. A randomized trial comparing postoperative adjuvant chemotherapy with cisplatin and 5-fluorouracil versus preoperative chemotherapy for localized advanced squamous cell carcinoma of the thoracic esophagus (JCOG9907). Ann Surg Oncol 2012;19:68-74.

16. Zheng Y, Li Y, Liu X, et al. Reevaluation of Neoadjuvant Chemotherapy for Esophageal Squamous Cell Carcinoma: A Meta-Analysis of Randomized Controlled Trials Over the Past 20 Years. Medicine (Baltimore) 2015;94:e1102.

17. Ajani JA, Ilson DH, Daugherty K, et al. Activity of taxol in patients with squamous cell carcinoma and adenocarcinoma of the esophagus. J Natl Cancer Inst 1994;86:1086-91.

18. Huang $\mathrm{X}$, Huang $\mathrm{G}$, Song $H$, et al. Preconditioning chemotherapy with paclitaxel and cisplatin enhances the antitumor activity of cytokine induced-killer cells in a murine lung carcinoma model. Int J Cancer 2011;129:648-58.

19. Hara H, Tahara M, Daiko H, et al. Phase II feasibility study of preoperative chemotherapy with docetaxel, cisplatin, and fluorouracil for esophageal squamous cell carcinoma. Cancer Sci 2013;104:1455-60.

(English Language Editor: L. Huleatt)

Cite this article as: Shang $\mathrm{X}$, Zhao G, Liang F, Zhang C, Zhang W, Liu L, Li R, Duan X, Ma Z, Yue J, Chen C, Meng B, Ren X, Jiang H. Safety and effectiveness of pembrolizumab combined with paclitaxel and cisplatin as neoadjuvant therapy followed by surgery for locally advanced resectable (stage III) esophageal squamous cell carcinoma: a study protocol for a prospective, single-arm, single-center, open-label, phase-II trial (Keystone-001). Ann Transl Med 2022;10(4):229. doi: $10.21037 /$ atm-22-513 